\title{
Obtaining the frequency characteristics of a generator, operating in a power system for tuning channels of stabilization of an automatic excitation controller
}

\author{
Nikita Filimonov ${ }^{1}$, and Alexey Yurganov, ${ }^{1, *}$ \\ ${ }^{1}$ Peter the Great St. Petersburg Polytechnic University, Polytechnicheskaya 29, St. Petersburg, \\ 195251, Russian Federation
}

\begin{abstract}
In this work we consider the issues concerning tuning of the channels of stabilization for the automatic excitation controller at a real object using the frequency response. We present a test procedure for the generator operating in the power system. Also, we give some recommendations for selecting the white-noise parameters, by which the frequency response is obtained. The results are considered on the example of four-machine scheme of the power system (the Kundur scheme).
\end{abstract}

\section{Introduction}

During the process of the excitation systems' commissioning there arises a problem for selection of the proper tuning for channels of the system stabilizer of the automatic excitation controller (AEC) and examination of the efficiency of the selected gain coefficients and time constants. In order to observe the efficiency for the introduction of the system stabilizer channels one should perform a strong excitation of the power system at various coefficients of the stabilizer channel strengthening. After this one should compare the results and select the optimum settings. At the power station such test works as short circuit or line cut-off are impossible, because it can cause loss of stability of the parallel operation of the power system. Nowadays the issues concerning the gain coefficients' tuning are solved using the mathematical simulation [1-6]. There exist the method for calculation the stability areas and the equal decaying areas using the D-splitting technique or the root analysis [7]. These methods require a detailed simulation of the objects of the power sys-tem, as well as knowledge of its scheme and detailed characteristics of the power equipment. Even in case of obtaining of all data and at creation of an accurate model there are no possibilities to prove efficiency of the gain-coefficients' tuning at a real object up to the moment of a breakdown or strong excitation in the power system. In this work we propose a procedure for tuning of the stabilizer channels based on the analysis of the frequency characteristics, which were obtained at the AEC unit while imposing the white noise.

\footnotetext{
*Corresponding author: aayurganov@mail.ru
} 


\section{Methods}

\subsection{The physical origin of the electromechanical oscillations}

The united electrical-power system comprises some resonance frequencies in each part of it, which occupy the range of the electromechanical oscillations from 0.1 to $5 \mathrm{~Hz}$. These frequencies are revealed at excitations of the power system and appear due to the energy exchange between the rotors of the synchronous generators. The oscillation frequency depends on some factors, but in general its value can be used for determination of the conditions, in which the power station or generator operates. The whole range of the electromechanical oscillations can be nominally divided into 3 parts:

1. Lower than $0.8 \mathrm{~Hz}$ is for intersystem oscillations. These are the oscillations between the systems which are electrically-remote distance one from another.

2. From 0.8 to $1.2 \mathrm{~Hz}$ is for intrasystem oscillations. The oscillations takes place within a certain system at an average electrical distance one from another.

3. Higher than $1.2 \mathrm{~Hz}$ is for intergroup oscillations. These oscillations take place between electrically-nearby generators. Usually these are generators of one power station.

The borders between groups may be blurred. Here they are presented figuratively. In case when there are some frequencies, the dominant one will be that which defines the stability of the parallel work.

\subsection{Definition of the frequencies of the electromechanical oscillations}

The frequencies of the electromechanical oscillations might be determined from the analysis of the parameters of the transient process after a serious breakage or excitation in a power system. From the physical point of view, receiving of the resonant frequencies is explained by the fact that during the incident the power system receives an excess power potentially at all possible frequencies, but the response and energy consumption will be performed at the resonance frequencies. Repetition of the same excitation with other con-troller settings allow one to compare the process oscillograms and see the operating effi-ciency of the stabilization channels. This can be called an active method for frequency definition of the electromechanical oscillations. But intentional creation even of a single strong excitation in a power system is unacceptable according to the reliability conditions of consumers' energy supply, so one needs another method.

\subsection{The white-noise technique}

The other method, in contrast to the previous one, might be called passive. It consists in creation of oscillations in a power system in a potentially possible frequency range of the electromechanical oscillations from $0.1 \mathrm{~Hz}$ to $5 \mathrm{~Hz}$ and recording of the generator output parameters in this mode. The idea is that at the resonance frequencies the power system will create a larger response. If we programmatically add noise of a low capacity, which comprises oscillation frequencies from $0.1 \mathrm{~Hz}$ to $5 \mathrm{~Hz}$ to an output signal of the automatic excitation controller, and record the parameters for some minutes, than we will observe its highest response at the resonance frequencies of the output parameters of the object. If we use the Fourier transform in a range of electromechanical frequencies to the oscillogram of active capacity during the noise action time, we will obtain amplitude-frequency spec-trum, in general case with some resonance peaks. By varying the time constants of the elements, and its gain coefficients and re-obtaining the frequency response after the noise imposing, one can see variation of the resonance peaks depending of the amplitude and frequency. Thus one can see the efficiency of the introduction of the system stabilizer channels at a real object. 


\subsection{Characteristics of the white noise}

One should advisedly select the noise characteristics. Noise will be added to the output signal of the automatic excitation controller. The mode should remain changeless, consequently, the noise mathematical expectation should be equal to zero. At pre-commissioning procedures at the generator operating stage in a power system the maxi-mum stator voltage deviation is acceptable to be from $-10 \%$ to $+10 \%$, so the noise dispersion should be chosen so that the generator voltage deviation is within these limits. The station auxiliaries and local consumers might be fed directly from the generator or from the generator voltage buses. So high oscillations of the stator voltage are not acceptable and it is necessary that the voltage oscillations at the generator buses do not exceed $5 \%$. Due to high response time of the generator at white noise imposing the voltage deviation won't reach these values. The limiting factor is that the thyristor angle of ignition should be in the range from 0 to 135 degrees so that the controller doesn't operate at the limitation mode. The minimal passing frequency will be $0.1 \mathrm{~Hz}$, and the maximum one will be $5 \mathrm{~Hz}$. The longer is the noise imposing time, the more statistical data will be obtained, the smoother the spectrum will be. At decomposition to the Fourier's series the spectrum will be non-uniform with dips and outliers at some frequencies, so it will be smoothened [8]. Basing on this, the sufficient imposing time appears to be 3-5 minutes. The most obvious generator parameter for the spectrum obtaining is the frequency, but its variation can be very small and the peaks could not be revealed in the spectrum. So, the best parameter for decomposition is the generator active capacity.

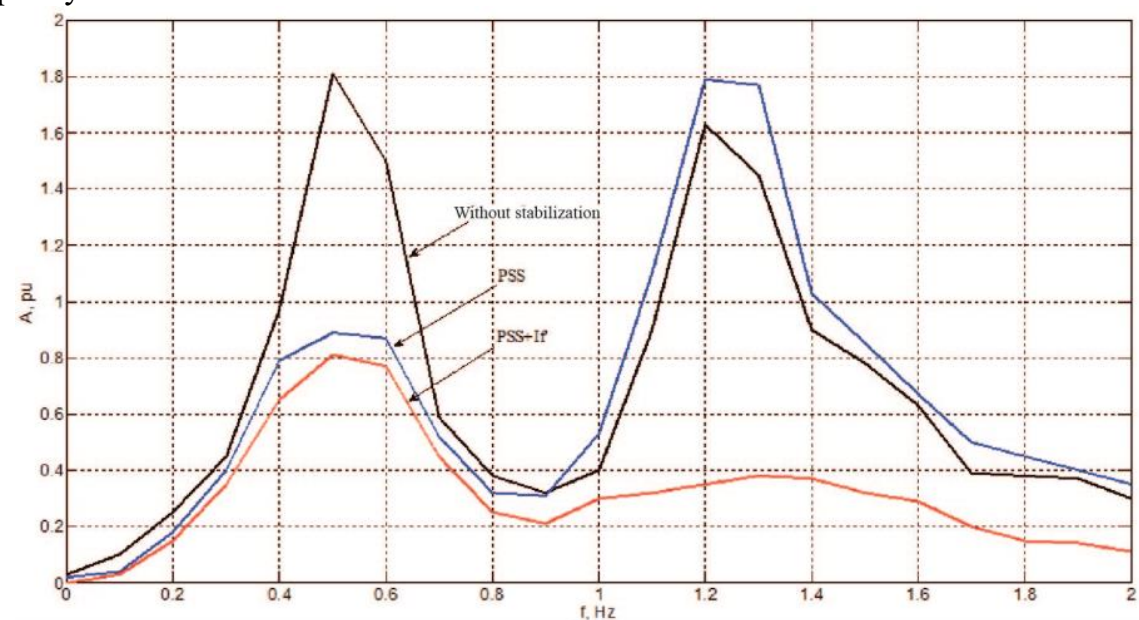

Fig. 1. Amplitude-frequency and phase-frequency characteristics of the filter (aperiodic element of the first order).

\section{Results and Discussion}

\subsection{Additional comments}

The required increment for frequency determination is in the range from 0.05 to $0.1 \mathrm{~Hz}$. The amount of counts for the fast Fourier transform is 4096 for this frequency range, which corresponds to the $0.061 \mathrm{~Hz}$ resolution. Despite the generator safety at the excitation noise mode, one should immediately stop the noise at sudden breakdown, shutdown or strong change of the operating mode. Withdrawal from the mode should be performed at the internal 
error of the exciting system, at obtaining the external discrete signal, at deviation the generator parameters for $10 \%$ from the initial value, besides the exciting voltage.

\subsection{The calculation results}

Fig. 1 presents frequency characteristics of the generator, which were obtained from decomposition of the generator active capacity into the Fourier's series at imposing the exciting noise for 3 minutes while its operation in a four-machine test scheme [9]. It was shown, that introduction of the stabilizer of the external motion leads to damping of the intersystem frequency.

\section{Conclusions}

In this work we present a procedure for tuning of the stabilizer coefficients at the power station by analysis of the frequency characteristics, obtained by fast Fourier transform to the generator active capacity at imposing the noise to the generator excitation winding. The advantage of this technique is its visibility and direct analysis of a real object, not its mathematical model.

\section{References}

1. A. Yurganov, V. Kozhevnikov Tuning of the excitation of the synchronic generators (1996).

2. A. Belyaev and D. Efremov. The suppression of slightly damped torsional oscillations in autonomous power systems, 1483 (7), (2017)

3. A. Lyamov, M. Makarova, S. Smolovik. Controllable shunt reactor deployment effect on power station stability indices, 105 (2), (2015)

4. Y. Vasiliev, P. Zegzhda, D. Zegzhda, Providing security for automated process control systems at hydropower engineering facilities, 948 (56), (2016)

5. P. Chernyaev, V. Chudny, S. Smolovik. Enhancement of required transmission capacity calculations for integrated power systems interconnections, 530-553, (2016).

6. N.A. Belyaev, N.V. Korovkin, V.S. Chudny, O.V. Frolov, Investigation of voltage level control in electric power systems (2015)

7. A.N. Belyaev, G.A. Pershikov, E.N. Popkov, S.V. Smolovik, V.S. Chudnyj, Electromagnetic transition processes in electrical power systems SPb.: SPbGPU, (2012).

8. M.L. Levinshtejn Operational calculus for the electromechanical problems [Operatsionnoe ischislenie v zadachah elektrotekhniki.] (1972).

9. P. Kundur Power system stability and control (1996). 